BULL. AUSTRAL. MATH. SOC.

VOL. 7 (1972), 377-385.

\title{
On the relation of a distributive lattice to its lattice of ideals
}

\section{Herbert S. Gaskill}

\begin{abstract}
In this note we examine the relationship of a distributive lattice to its lattice of ideals. Our main result is that a distributive lattice and its lattice of ideals share exactly the same collection of finite sublattices. In addition we give a related result characterizing those finite distributive lattices $L$ which can be embedded in a lattice ' $L$ ' whenever they can be embedded in its lattice of ideals $T\left(L^{\prime}\right)$.
\end{abstract}

In this note our main result is the following: if $L$ is a distributive lattice and $T(L)$ its lattice of ideals, then $L$ and $T(L)$ have the same collection of finite substructures. In addition we give a characterization of those finite distributive lattices $L$ for which if $L^{\prime}$ is any lattice and $L$ is embeddable in $T\left(L^{\prime}\right)$ then $L$ can be embedded in $L^{\prime}$.

\section{Preliminaries}

In general we follow the notation of Grätzer [3]. By a lattice we mean a structure $\langle L ;+, \cdot\rangle$ where + and - are binary, associative, commutative, idempotent, and related by,

$$
x+(y \cdot x)=x \text { and } x(x+y)=x .
$$

We often omit the - If $x+y=x$, then $x y=y$, and we then write $y \leq x$. By an ideal of a lattice $L$ we mean a non-empty subset $I$ of $L$

Received 14 June 1972. Much of this research stems from work on the author's PhD thesis carried out under Professor A.H. Lachlan, to whose stimulation the author makes grateful acknowledgement. 
such that if $a$ and $b \in I$ and $c \leq a+b$ then $c \in I$. We will denote the collection of all ideals of $L$ by $I(L)$. It is well known that the structure $T(L)=\langle I(L) ;+, n\rangle$ is a lattice where $n$ is set theoretic intersection and + is defined by

$$
I_{1}+I_{2}=\left\{a: a \in L \text { and } \exists b_{1} \in I_{1}, 3 b_{2} \in I_{2} \text { and } a \leq b_{1}+b_{2}\right\} \text {. }
$$

If $I_{0} \in I(L)$ and for some $a \in L$,

$$
I_{0}=\{b: b \in L \text { and } b \leq a\} \text {, }
$$

then we say $I_{0}$ is the principal ideal generated by $a$. We will denote the principal ideal generated by $a$ by $\bar{a}$. Lastly a lattice is distributive if it satisfies

$$
x(y+z)=x y+x z .
$$

1.

In this section we give a complete characterization of those finite distributive lattices $L$ which satisfy the condition that whenever $L$ is embeddable in $T\left(L^{\prime}\right)$ then $L$ can be embedded in $L^{\prime}$. We have termed such lattices weakly transferable. The problem of characterizing weakly transferable lattices was first raised by Grätzer in [4, p. 207], and at that time he pointed out the following:

LEMMA 1. If $L$ is any finite lattice and $L$ has a point which is both join and meet reducible, then $L$ is weakly transferable.

We shall show that if $L$ is a finite distributive lattice and no point of $L$ is both join and meet reducible, then $L$ is weakly transferable. In fact we show an even stronger result. We say that a finite lattice is transferable if whenever $\phi$ embeds $L$ in $T\left(L^{\prime}\right)$ there is a $\psi$ embedding $L$ in $L^{\prime}$ such that $x \psi \in y \phi$ if and only if $x \leq y$. Thus the embedding of $L$ in $L^{\prime}$ relates in a substantial way to the embedding of $L$ in $T\left(L^{\prime}\right)$. For the remainder of this paper $L=\langle L ;+, \cdot\rangle$ denotes a fixed finite distributive lattice.

THEOREM 1. If no point of $L$ is both join and meet reducible, then $L$ is transferable.

Before proceeding with the proof of this result we shall need some 
information about the structure of finite distributive lattices.

LEMMA 2 [1, p. 58]. If $L$ is a finite distmibutive lattice, then every element has a unique representation as a join of a join-irredundant set of join irreducibles.

Using Lemma 2, for each $x \in L$, let $J_{x}$ denote that unique join irredundant set of join irreducibles satisfying $\sum J_{x}=x$. As an immediate consequence of the lemma we obtain:

LEMMA 3. If $\phi$ is any map of $L$ into a lattice $L^{\prime}$ such that $\phi$ is order preserving on the set of join irreducibles and such that $x \phi=\sum\left(J_{x} \phi\right)$ for each $x \in L$, then $\phi$ is a join homomorphism.

Proof. This is immediate from the fact that Lemma 2 tells us that every join irredundant set of join irreducibles sums to a unique element. For greater detail see [1].

Proof of Theorem 1. Let $\phi$ be the embedding of $L$ in $T\left(L^{\prime}\right)$ where

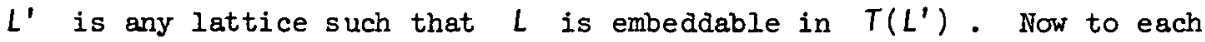
$x \in L$ choose an $x_{0} \in L^{\prime}$ such that $x_{0} \in y \phi$ if and only if $x \leq y$. Such choices are possible since $\phi$ is an embedding. Note that if $x \phi$ is principal then $x \phi=\bar{a}$ for some $a \in L$ and we may take $x_{0}=a$. It is now clear that if $\psi$ is a homomorphism of $L$ into $L^{\prime}$ such that $x_{0} \leq x \psi \in x \phi$ then $\psi$ is one-to-one. Further it is easily seen that for each $x \in L$ we can choose a $\psi_{x}$ such that $\psi_{x}$ is defined exactly on the set of join irreducibles of $L, \psi_{x}$ is order preserving, for each join irreducible $y, y_{0} \leq y \psi_{x} \in y \phi$, and such that $x_{0} \leq \sum\left(J \psi_{x}\right)$. We then define $\psi^{*}$ by

$$
y \psi^{*}= \begin{cases}\left\{\left\{y \psi_{x}: x \in L\right\}\right. & \text { if } y \text { is join irreducible, } \\ {\left[\left(J_{y} \psi^{*}\right)\right.} & \text { if } y \text { is join reducible. }\end{cases}
$$

It is clear that $\psi^{*}$ is a join embedding and that $x \psi^{*} \in y \phi$ if and only if $x \leq y$. To complete the proof we define $\psi$ by

$$
x \psi=\prod\left(M_{x} \psi^{*}\right)
$$


where $M_{x}$ is the unique meet irredundant meet representation of $x$ as a meet of meet irreducibles given by the dual of Lemma 2. Since $x \psi^{*} \leq x \psi \in x \phi$, we have that $\psi$ is a meet isomorphism satisfying $x \psi \leq y \phi$ if and only if $x \leq y$. Now we assert that $\psi$ is a lattice isomorphism. It is well known that in any distributive lattice, if $z \leq x+y$ then there exists $x_{1} \leq x$ and $y_{1} \leq y$ such that $x_{1}+y_{1}=z$. Similarly the dual of this result is valid. Consider $a, b, c$ and $d \in L$ such that $c d \leq a+b$ where $a+b$ is a proper join and $c d$ a proper meet. Since $c d$ is not join reducible we must have $c d \leq a$ or $c d \leq b$. Since $a+b$ is not meet reducible we must have either $c \leq a+b$ or $d \leq a+b$. Now $\psi$ is a meet isomorphism whence $\psi$ preserves the valid inequality of $c d \leq a$ and $c d \leq b$. Without loss of generality assume $c \leq a+b$. There are two cases.

Case 1. $c$ is meet irreducible. Since $c \psi=c \psi^{*}$ for this case, we obtain

$$
c \psi=c \psi^{*} \leq a \psi^{*}+b \psi^{*} \leq a \psi+b \psi
$$

since $\psi^{*}$ is a join isomorphism, which completes Case $I$.

Case 2. $c$ is meet reducible. For this case, $c$ is join irreducible whence for some $x_{0} \in J_{a+b}, c \leq x_{0}$ by Lemma 2 . Since $\psi$ is an order isomorphism and $J_{a+b} \subseteq J_{a} \cup J_{b}$, we have

$$
c \psi \leq x_{0} \psi \leq a \psi+b \psi
$$

which completes Case 2 .

It follows that for arbitrary $a, b, c$ and $d \in L$, whenever $c d \leq a+b$ then $(a \psi)(d \psi) \leq a \psi+b \psi$. It is immediate that $\psi$ is ar. isomorphism, and this completes the proof of Theorem 1 .

Distributive lattices in which no point is both join and meet reducible have a particularly nice structure. This description was first obtained by Galvin and Jónsson [2] and for the sake of completeness we give this description. Given two non-empty subsets $L_{1}$ and $L_{2}$ of $L$, we will write $L_{1} \leq L_{2}$ if and only if $L_{1}=L_{2}$ or for each $x \in L_{1}$ and $y \in L_{2}, x<y, L$ is said to be linearly indecomposable if there do not 
exist $L_{1}$ and $L_{2}$ such that $L_{1}<L_{2}$ and $L_{1} \cup L_{2}=L$. It is straight forward that $L$ is the union of a unique finite linearly ordered family $C_{L}$ of linearly indecomposable lattices.

THEOREM 2 (Galvin and Jónsson). If no point of $L$ is both join and meet reducible and $L_{1} \in C_{L}$ then either $L_{1}$ is the 1 element lattice or $L_{1}$ is the 8 element boolean lattice or $L_{1}$ is a direct product of the 2 element chain with a finite chain having 2 or more elements.

2.

In this section we show that if $L^{\prime}$ is a distributive lattice then $L^{\prime}$ and $T\left(L^{\prime}\right)$ have exactly the same collection of finite sublattices. For the remainder, let $L^{\prime}$ be a fixed infinite distributive lattice. Let $L$ be a fixed finite distributive lattice with $\phi$ embeding $L$ in $T\left(L^{\prime}\right)$. Further let $\psi^{*}$ and $\psi$ be obtained as in the proof of Theorem $I$. With $J_{x}$ and $M_{x}$ as before, it is easily seen that if for each $x \in L$, $\sum\left(J_{x} \psi\right)=\prod\left(M_{x} \psi\right)$, then $\psi$ is a lattice isomorphism. Further from the definition of $\psi$ we have $\sum\left(J_{x} \psi\right) \leq \prod\left(M_{x} \psi\right)$. Thus if $\psi$ is not a lattice isomorphism then there is an $x$ such that

$$
\sum\left(J_{x} \psi\right)<\prod\left(M_{x} \psi\right)
$$

Such an $x$ is clearly meet and join reducible.

LEMMA 4. Let $[L \psi]$ be the lattice closure of $L \psi$ in $L^{\prime}$. Then if $y \in[L \psi] \sim L \psi$, there is an $x_{y} \in L$ such that $\sum\left(J_{x_{y}} \psi\right) \leq y<\prod\left(M_{x_{y}} \psi\right)$.

Proof. We define sets $K_{0}, \ldots, K_{n}, \ldots$ as follows: $K_{0}=L \psi$, $K_{2 i+1}$ is the join closure of $K_{2 i}$ and $K_{2 i+2}$ is the meet closure of $K_{2 i+1}$. Now for some $n_{0} \in \omega,[L \psi]=K_{n_{0}}$ since $L^{\prime}$ is distributive. Suppose that for each $j$ such that $0 \leq j<n$, if $j \in K_{j} \sim L \psi$ then the lemma is satisfied. Consider $y \in K_{n} \sim L \psi$. 
Case 1. $n=2 n+1 \geq 1$. Now if $y \in K_{n} \sim K_{n-1}$, then there is a set of elements $J_{y} \subseteq K_{n-1}$ which is join irredundant and such that $\sum J_{y}=y$. Now for each $z \in J_{y}$ there is an $x_{z} \in L$ such that $\sum\left(J_{x_{z}} \psi\right) \leq z \leq \prod\left(M_{x_{z}} \psi\right)$. Let

$$
x_{y}=\sum\left\{U\left\{J_{x_{z}}: z \in J_{y}\right\}\right) .
$$

Now $\sum J_{x_{y}}=\prod M_{x y}$, whence for each $z \in J_{y}$ we have $z<\prod\left(M_{x_{z}} \psi\right) \leq \prod\left(M_{x_{y}} \psi\right)$, whence we conclude that

$$
\sum\left(J_{x_{y}} \psi\right) \leq y<T\left(M_{x_{y}} \psi\right)
$$

as desired. This completes Case 1.

Case 2. $n=2 m+2 \geq 2$. The treatment of this case is similar to that of Case 1, and we omit the details.

Observe that Lemma 4 allows us to draw the conclusion that, if $x \in L$ is join irreducible, then $x \psi$ is join irreducible in $([L \psi] ;+, \cdot)$.

LEMMA 5. Let a be a fixed maximal member of $L$ such that $\sum\left(J_{a} \psi\right)<\prod\left(M_{a} \psi\right)$. Then for alt $x \in L$ either $x \leq a$ or $a \leq x$.

Proof. As noted earlier, $a$ must be both join and meet reducible. Suppose for the sake of contradiction that there is a $d \in L$ with $a \neq d$ and $d \neq a$. Now $\sum\left(J_{a} \psi\right)<\prod\left(M_{a} \psi\right)$ and $\sum\left(J_{a} \psi\right) \in[L \psi]$. Let

$$
H=\{x: x \in L, \quad x \text { is meet irreducible and } x \neq a\} .
$$

Then for each $y \in L$ either $y \leq \prod H$ or $\prod H \leq y$. To see this we note that for any meet irreducible $c$, if $c \leq a$ then $c \leq \prod H$ by the dual of Lemma 2. Further by assumption $\prod H<a$. Now either $\prod H$ is join irreducible or there is a foin irreducible $c$ such that $\prod H<c<a$. Hence fix $c$ such that $c$ is join irreducible and 
$\prod H \leq c<a$. Then $c \psi=\sum\left(J_{c} \phi\right)=\prod M_{c} \psi$, whence

$$
\prod\left(M_{c} \psi\right)<\sum\left(J_{a} \psi\right)<\prod\left(M_{a} \psi\right)
$$

For convenience let $\left\{\left(J_{a} \psi\right)=b\right.$ and $M_{c}=\left\{b_{0}, \ldots, b_{m-1}\right\}$. Since $a \psi^{*} \leq b<\prod\left(M_{a} \psi\right) \in a \phi$, if for some $b_{i}, b_{i} \psi \leq b$ then we can conclude that $b_{i} \psi \in a \phi$ contrary to hypothesis. Thus for each $b_{i}, b_{i} \neq b$. Now by distributivity we obtain

$$
b=b+\prod_{i \in m}\left(b_{i} \psi\right)=\prod_{c \in m}\left(b+b_{i} \psi\right)
$$

Fix an $i$ and let $c_{i} \in L$ be such that

$$
\sum\left(J_{c_{i}} \psi\right) \leq\left(b+b_{i} \psi\right) \leq \prod\left(M_{c_{i}} \psi\right) \text {. }
$$

We immediately conclude that

$$
a \psi^{*} \leq b \leq \prod\left(M_{c_{i}} \psi\right) \in c_{i} \phi,
$$

whence $a \leq c_{i}$ by definition of $\psi^{*}$. By the maximality of a we obtain $c_{i} \psi=b+b_{i} \psi$ whence

$$
b<a \psi \leq \prod_{i \in m}\left(c_{i} \psi\right)=b
$$

which is absurd. Thus for all $x \in L$ either $a \leq x$ or $x \leq a$.

LEMMA 6. Let $L$ and $L^{\prime}$ be as before with $\phi$ embedding $L$ in $T(L)$. Let 1 be the greatest element of $L$. If $a \in I \phi$ such that for all $y \in L \sim\{1\}, a k y \phi$ then the map $\phi^{\prime}$ defined by $x \phi^{\prime}=\bar{a} \cap x \phi$ is an isomorphism.

Proof. That $\phi^{\prime}$ is a meet isomorphism is obvious. Let $b \in L$. We must show that if $b$ is join reducible with $J_{b}=\left\{b_{0}, \ldots, b_{m-1}\right\}$ and $c \in b \phi^{\prime}$ then we can choose $c_{i} \in b_{i} \phi^{\prime}$ such that $\sum c_{i} \geq c$. But this is obvious, whence $\phi^{\prime}$ is an isomorphism.

THEOREM 3. Let $L$ and $L^{\prime}$ be as before. If $L$ can be embedded 
in $T\left(L^{\prime}\right)$ then $L$ can be embedded in $L$

Proof. Let $b_{0}, \ldots, b_{m-1}$ be a list in descerding order of those elements of $L$ excluding 0 and $l$ which satisfy for all $x \in L, x \leq y$ or $y \leq x$. Let $\psi$ be obtained as in Theorem 1. Then if $\psi$ is not the desired isomorphism it fails for a maximal $b_{j_{0}}$ where for each $x \in L$ if $b_{j_{0}}<x$ then $\sum\left(J_{x} \psi\right)=\prod\left(M_{x} \psi\right)$. We define a new isomorphism $\phi_{1}: L+I\left(L^{\prime}\right)$ by:

$$
x \phi=\left\{\begin{array}{lll}
x \psi & \text { if } b_{j_{0}} \leq x, \\
\overline{x_{j_{0}} \psi} \cap x \phi & \text { if } x<b_{j_{0}} .
\end{array}\right.
$$

By procedures outlined in the proof of Theorem 1 we obtain a $\psi_{1}$ such that for all $x \in L, x \psi_{1} \in y \phi_{1}$ if and only if $x \leq y$. Further we may also require that $x \psi_{1}=x \psi$ for each $x$ such that $b_{j_{0}} \leq x$, and in addition that $x \psi_{1} \leq y \phi$ if and only if $x \leq y$. Since if $\psi_{1}$ is not the desired isomorphism, its failure occurs at $b_{j_{1}}<b_{j_{0}}$, we are done since the set of $b_{j}$ 's is finite. This concludes the proof and the note.

\section{References}

[1] Garrett Birkhoff, Lattice theory, 3rd ed. (Colloquium Publ. 25, Amer. Math. Soc., Providence, Rhode Island, 1967).

[2] Fred Galvin and Bjarni Jónsson, "Distributive sublattices of a free lattice", Canad. J. Math. 13 (1961), 265-272.

[3] George Grätzer, Universal algebra (Van Nostrand, Princeton, New Jersey; Toronto; London; Melbourne; 1968). 
[4] George Grätzer, "Universal algebra", Trends in Zattice theory

(Sympos., US Naval Academy, Annapolis, Md., 1966, 173-210. Van Nostrand Reinhold Co., New York; Toronto, Ontario; London; 1970).

\footnotetext{
Department of Mathematics,

Simon Fraser University,

Burnaby,

British Columbia,

Canada.
} 